\title{
THE CESÀRO MEANS AND STRONG LAWS OF LARGE NUMBERS FOR ORTHOGONAL SEQUENCES IN VON NEUMANN ALGEBRAS
}

\author{
EWA HENSZ \\ (Communicated by Palle E. T. Jorgensen)
}

\begin{abstract}
The extension of Moricz's strong law of large numbers to the von Neumann algebras context is given.
\end{abstract}

\section{INTRODUCTION}

Let $(\Omega, \mathscr{F}, \mu)$ be a probability space and let $\left(X_{k}: k \geq 1\right)$ be an orthogonal sequence of random variables with finite second moments, i.e., $E X_{k}^{2}<\infty$, $(k \geq 1)$ and $E X_{k} X_{l}=0,(k, l \geq 1, k \neq l)$.

The most important result concerning the almost sure convergence of series of pairwise orthogonal random variables is the well-known Rademacher-Menshov theorem. This theorem asserts that if

$$
\sum_{k=1}^{\infty}\left(\log ^{2} k\right) E X_{k}^{2}<\infty
$$

then the series $\sum_{k=1}^{\infty} X_{k}$ is convergent almost surely [8, 5], (cf. also [4, p. 164]). (In this paper the logarithms are of base 2.) This theorem has a simple consequence (via the Kronecker lemma) in the form of the following strong law of large numbers.

If

$$
\sum_{k=1}^{\infty} \frac{\log ^{2} k}{k^{2}} E X_{k}^{2}<\infty,
$$

then the first arithmetic means

$$
Z_{n}=\frac{1}{n} \sum_{k=1}^{n} X_{k}, \quad(n \geq 1),
$$

converge to 0 almost surely.

Received by the editors March 24, 1989 and, in revised form, December 17, 1989.

1980 Mathematics Subject Classification (1985 Revision). Primary 46L50; Secondary 60F15.

Key words and phrases. von Neumann algebra, faithful normal state, almost uniform convergence, orthogonal sequences, Cesàro means, strong laws of large numbers. 
The first means $\left(Z_{n}\right)$ of the sequence $\left(X_{n}\right)$ are the special case (when $\alpha=0$ ) of the Cesàro means of order $\alpha$ ( $\alpha$ real number)

$$
T_{n}^{\alpha}=\frac{1}{n A_{n-1}^{\alpha}} \sum_{k=1}^{n} A_{n-k}^{\alpha} X_{k}, \quad(n \geq 1),
$$

where $A_{0}^{\alpha}=1$ and $A_{m}^{\alpha}=\left(\begin{array}{c}a+m \\ m\end{array}\right)=((\alpha+1) \cdots(\alpha+m)) / m$ ! for $m \geq 1$.

In particular, when $\alpha=1$, we obtain

$$
T_{n}=T_{n}^{1}=\frac{1}{n} \sum_{k=1}^{n}\left(1-\frac{k-1}{n}\right) X_{k}, \quad(n \geq 1) .
$$

Recently, in $[6,7]$ Moricz has shown that if

$$
\sum_{k=1}^{\infty} \frac{1}{k^{2}} E X_{k}^{2}<\infty
$$

then the means $T_{n}$ converge to 0 almost surely.

The above result can obviously be treated as a strong law of large numbers as well.

On the other hand, a great deal of work has been done lately to generalize various strong limit theorems to the von Neumann algebras context (cf. Jajte [2]).

Roughly speaking, in this case, the algebra $L_{\infty}$ over a probability space $(\Omega, \mathscr{F}, \mu)$ is replaced by an arbitrary von Neumann algebra $M$ of operators acting in a Hilbert space. The role of the measure $\mu$, more exactly, the integral with respect to $\mu$, is played by a normal faithful state $\Phi$ on $M$. The classical Egorov theorem makes it possible to introduce the analogue of the almost sure convergence. The first and simplest version is known as the almost uniform convergence of operators.

Many classical results have been extended for traces, i.e., for finite von Neumann algebras. However, for states, the proofs are more complicated and need some new approach (because, in general, the states are not subadditive on the lattice of projections in a von Neumann algebra). One of the possibilities that is very useful, in particular, in proving strong laws of large numbers, is a powerful method of operator estimation which is based on a special case of the noncommutative maximal ergodic theorem of Goldstein [1].

Recently, in [3] Jajte has extended the Rademacher-Menshov strong law of large numbers to the von Neumann algebras case.

The aim of this paper is to give the analogue of Moricz's law of large numbers in this case.

It is worthwhile noticing that the Rademacher-Menshov theorem does not hold in this context because even in the classical case the limit of bounded random variables can be unbounded. For this reason we can only consider the extension of laws of large numbers just formulated. 


\section{Preliminaries}

Let us begin with some notations and definitions. Throughout the paper, $M$ will denote a $\sigma$-finite von Neumann algebra of operators with a faithful normal state $\Phi$. For a projection $p \in M$, always $p^{\perp}=1-p$, and $|x|=\left(x^{*} x\right)^{1 / 2}$ for $x \in M$. We will often use the inequality

$$
\left|\sum_{k=1}^{n} x_{k}\right|^{2} \leq n \sum_{k=1}^{n}\left|x_{k}\right|^{2},
$$

where $x_{1}, \ldots, x_{n} \in M$, which is implied by the Schwartz inequality $x^{*} y+$ $y^{*} x \leq x^{*} x+y^{*} y$ for $x, y \in M$.

We recall that a sequence $\left(x_{n}\right)$ in $M$ converges almost uniformly to $x \in M$, $\left(x_{n} \rightarrow x\right.$ a.u. $)$ if, for each $\varepsilon>0$, there exists a projection $p \in M$ with $\Phi\left(p^{\perp}\right)<\varepsilon$ and such that $\left\|\left(x_{n}-x\right) p\right\| \rightarrow 0$ as $n \rightarrow \infty$.

We say that a sequence $\left(x_{n}\right)$ in $M$ is orthogonal (relative to the state $\Phi$ ) if $\Phi\left(x_{k}^{*} x_{l}\right)=0$ for $k \neq l$.

In the end, we formulate the theorem of Goldstein which is a basic tool in our consideration.

Theorem 0 . Let $\left(a_{n}\right)$ be a sequence of positive operators in $M$ and $\left(\varepsilon_{n}\right)$ a sequence of positive numbers. Then there exists a projection $p \in M$ with

$$
\Phi\left(p^{\perp}\right) \leq 2 \sum_{k=1}^{\infty} \varepsilon_{k}^{-1} \Phi\left(a_{k}\right)
$$

and such that $\left\|p a_{n} p\right\|<2 \varepsilon_{n}$ for all $n \geq 1$.

\section{Main Result}

For a sequence of $\left(x_{k}\right)$ in $M$ let us put

$$
\begin{aligned}
z_{n} & =\frac{1}{n} \sum_{k=1}^{n} x_{k}, \quad(n \geq 1), \\
t_{n} & =\frac{1}{n} \sum_{k=1}^{n}\left(1-\frac{k-1}{n}\right) x_{k}, \quad(n \geq 1) .
\end{aligned}
$$

Now, we extend Moricz's law of large numbers.

Theorem. Let $\left(x_{n}\right)$ be an orthogonal sequence in a von Neumann algebra $M$ with a faithful normal state $\Phi$. If

$$
\sum_{k=1}^{\infty} \frac{1}{k^{2}} \Phi\left(\left|x_{k}\right|^{2}\right)<\infty
$$

then the Cesàro means $t_{n}=\frac{1}{n} \sum_{k=1}^{n}(1-(k-1) / n) x_{k} \rightarrow 0$ a.u. 
Proof. At the beginning, we adopt the idea which is well known in the theory of orthogonal series; namely, the "dyadic" estimation. For every projection $p \in M$ and $2^{n}<m \leq 2^{n+1}$ we have

$$
\begin{aligned}
\left\|t_{m} p\right\|^{2} & =\left\|\left(t_{m}-t_{2^{n}}\right) p+t_{2^{n}} p\right\|^{2} \\
& \leq 2\left\|\left(t_{m}-t_{2^{n}}\right) p\right\|^{2}+2\left\|t_{2^{n}} p\right\|^{2} \\
& \leq 2\left\|p\left|t_{m}-t_{2^{n}}\right|^{2} p\right\|+2\left\|p\left|t_{2^{n}}\right|^{2} p\right\| .
\end{aligned}
$$

Therefore, in order to show that $t_{m} \rightarrow 0$ a.u. when $m \rightarrow \infty$, it is enough to find, for every $\varepsilon>0$, a projection $p \in M$ with $\Phi\left(p^{\perp}\right)<\varepsilon$ and a sequence $\left(\delta_{n}\right)$ of positive numbers with $\delta_{n} \rightarrow 0$ such that for $n=1,2, \ldots$,

$$
\left\|p\left|t_{2^{n}}\right|^{2} p\right\|<\delta_{n},\left\|p\left|t_{m}-t_{2^{n}}\right|^{2} p\right\|<\delta_{n}, \quad 2^{n}<m \leq 2^{n+1} .
$$

In the sequel, we are going to follow the general idea of Moricz, so the proof will be done in several steps. The first two steps are similar to the classical case.

(i) First, we prove that

$$
\sum_{n=0}^{\infty} \Phi\left(\left|z_{2^{n}}\right|^{2}\right)<\infty .
$$

In fact, by orthogonality, we have

$$
\Phi\left(\left|z_{n}\right|^{2}\right)=\frac{1}{n^{2}} \sum_{k=1}^{n} \Phi\left(\left|x_{k}\right|^{2}\right) .
$$

Thus,

$$
\begin{aligned}
\sum_{n=0}^{\infty} \Phi\left(\left|z_{2^{n}}\right|^{2}\right) & =\sum_{n=0}^{\infty} \frac{1}{2^{2 n}} \sum_{k=1}^{2^{n}} \Phi\left(\left|x_{k}\right|^{2}\right) \\
& =\sum_{k=1}^{n} \Phi\left(\left|z_{k}\right|^{2}\right) \sum_{\left\{n: 2^{n} \geq k\right\}} \frac{1}{2^{2 n}} \\
& \leq \text { const } \sum_{k=1}^{\infty} \frac{1}{k^{2}} \Phi\left(\left|x_{k}\right|^{2}\right)<\infty
\end{aligned}
$$

(ii) Our next step is to prove that

$$
\sum_{n=0}^{\infty} \Phi\left(\left|z_{2^{n}}-t_{2^{n}}\right|^{2}\right)<\infty .
$$

Since

$$
z_{n}-t_{n}=\frac{1}{n^{2}} \sum_{k=1}^{n}(k-1) x_{k}
$$


a simple calculation gives that

$$
\begin{aligned}
\sum_{n=0}^{\infty} \Phi\left(\left|z_{2^{n}}-t_{2^{n}}\right|^{2}\right. & =\sum_{n=0}^{\infty} \frac{1}{2^{4 n}} \sum_{k=2}^{2^{n}}(k-1)^{2} \Phi\left(\left|x_{k}\right|^{2}\right) \\
& =\sum_{k=2}^{\infty}(k-1)^{2} \Phi\left(\left|x_{k}\right|^{2}\right) \sum_{\left\{n: 2^{n} \geq k\right\}} \frac{1}{2^{4 n}} \\
& \leq \text { const } \sum_{k=1}^{\infty} \frac{1}{k^{2}} \Phi\left(\left|x_{k}\right|^{2}\right)<\infty .
\end{aligned}
$$

By (1) and (2) we get

$$
\sum_{n=0}^{\infty} \Phi\left(\left|t_{2^{n}}\right|^{2}\right)<\infty
$$

(iii) Now, we shall estimate the operators

$$
t_{m}-t_{2^{n}} \text { for } 2^{n}<m \leq 2^{n+1} \text {. }
$$

We have

$$
t_{m}-t_{2^{n}}=\sum_{j=2^{n}+1}^{m}\left(t_{j}-t_{j-1}\right)
$$

thus, we get

$$
\left|t_{m}-t_{2^{n}}\right|^{2} \leq\left(m-2^{n}\right) \sum_{j=2^{n}+1}^{m}\left|t_{j}-t_{j-1}\right|^{2}
$$

Putting

$$
D_{n}=2^{n} \sum_{j=2^{n}+1}^{2^{n+1}}\left|t_{j}-t_{j-1}\right|^{2}, \quad(n \geq 0),
$$

we obtain $D_{n} \in M, D_{n} \geq 0$ and

$$
\left|t_{m}-t_{2^{n}}\right|^{2} \leq D_{n} \quad \text { for } 2^{n}<m \leq 2^{n+1}
$$

Now, we show that

$$
\sum_{n=0}^{\infty} \Phi\left(D_{n}\right)<\infty
$$

By the equality

$$
t_{j}-t_{j-1}=\sum_{k=1}^{j}\left(\frac{(k-1)(2 j-1)}{j^{2}(j-1)^{2}}-\frac{1}{j(j-1)}\right) x_{k}, \quad j \geq 2,
$$

we obtain

$$
\begin{aligned}
\Phi\left(\left|t_{j}-t_{j-1}\right|^{2}\right) & =\sum_{k=1}^{j}\left(\frac{(k-1)(2 j-1)}{j^{2}(j-1)^{2}}-\frac{1}{j(j-1)}\right)^{2} \Phi\left(\left|x_{k}\right|^{2}\right) \\
& \leq \sum_{k=1}^{j}\left(\frac{(k-1)^{2}(2 j-1)^{2}}{j^{4}(j-1)^{4}}+\frac{1}{j^{2}(j-1)^{2}}\right) \Phi\left(\left|x_{k}\right|^{2}\right)
\end{aligned}
$$


and thus

$$
\begin{aligned}
\Phi\left(D_{n}\right)= & 2^{n} \sum_{j=2^{n}+1}^{2^{n+1}} \Phi\left(\left|t_{j}-t_{j-1}\right|^{2}\right) \\
\leq & 2^{n} \sum_{j=2^{n}+1}^{2^{n+1}} \sum_{k=1}^{j}\left(\frac{(k-1)^{2}(2 j-1)^{2}}{j^{4}(j-1)^{4}}+\frac{1}{j^{2}(j-1)^{2}}\right) \Phi\left(\left|x_{k}\right|^{2}\right) \\
\leq & 5 \cdot 2^{n} \sum_{j=2^{n}+1}^{2^{n+1}} \sum_{k=1}^{j} \frac{1}{j^{2}(j-1)^{2}} \Phi\left(\left|x_{k}\right|^{2}\right) \\
= & 5 \cdot 2^{n} \sum_{k=1}^{2^{n}} \Phi\left(\left|x_{k}\right|^{2}\right) \sum_{j=2^{n}+1}^{2^{n+1}} \frac{1}{j^{2}(j-1)^{2}} \\
& +5 \cdot 2^{n} \sum_{k=2^{n}+1}^{2^{n+1}} \Phi\left(\left|x_{k}\right|^{2}\right) \sum_{j=k}^{2^{n+1}} \frac{1}{j^{2}(j-1)^{2}} \\
\leq & 5 \cdot 2^{n} \sum_{k=1}^{2^{n+1}} \Phi\left(\left|x_{k}\right|^{2}\right) \sum_{j=2^{n}+1}^{2^{n+1}} \frac{1}{j^{2}(j-1)^{2}} \\
\leq & \frac{5}{\left(2^{n}+1\right)^{2}} \sum_{k=1}^{2^{n+1}} \Phi\left(\left|x_{k}\right|^{2}\right) .
\end{aligned}
$$

Hence, we get

$$
\begin{aligned}
\sum_{n=0}^{\infty} \Phi\left(D_{n}\right) & \leq 5 \sum_{n=0}^{\infty} \frac{1}{\left(2^{n}+1\right)^{2}} \sum_{k=1}^{2^{n+1}} \Phi\left(\left|x_{k}\right|^{2}\right) \\
& =5 \sum_{k=1}^{\infty} \Phi\left(\left|x_{k}\right|^{2}\right) \quad \sum_{\left\{n: 2^{n+1} \geq k\right\}} \frac{1}{\left(2^{n}+1\right)^{2}} \\
& \leq \text { const } \sum_{k=1}^{\infty} \frac{1}{k^{2}} \Phi\left(\left|x_{k}\right|^{2}\right)<\infty .
\end{aligned}
$$

(iv) Let $\varepsilon>0$ be given. By (3) and (5) we can find a sequence of $\left(\varepsilon_{n}\right)$ of positive numbers such that $\varepsilon_{n} \rightarrow 0$ and

$$
\sum_{n=0}^{\infty} \varepsilon_{n}^{-1} \Phi\left(\left|t_{2^{n}}\right|^{2}+D_{n}\right)<\frac{\varepsilon}{2} .
$$

By Theorem 0 there is a projection $p \in M$ with $\Phi\left(p^{\perp}\right)<\varepsilon$ and such that

$$
\begin{aligned}
& \left\|p\left|t_{2^{n}}\right|^{2} p\right\|<2 \varepsilon_{n}, \\
& \left\|p D_{n} p\right\|<2 \varepsilon_{n}, \quad n \geq 0 .
\end{aligned}
$$

Thus, we have by (4)

$$
\left\|p\left|t_{m}-t_{n}\right|^{2} p\right\| \leq\left\|p D_{n} p\right\|<2 \varepsilon_{n}
$$

which according to $(0)$, ends the proof. 


\section{REFERENCES}

1. M. S. Goldstein Theorems in almost everywhere convergence in von Neumann algebras, J. Operator Theory 6 (1981), 233-311. (Russian)

2. R. Jajte Strong limit theorems in noncommutative probability, Lecture Notes in Math., vol. 1110, Springer-Verlag, Berlin, Heidelberg, New York, and Tokyo, 1985.

3. __ Strong limit theorems for orthogonal sequences in von Neumann algebras, Proc. Amer. Maî. Soc. 94 (1985), 229-236.

4. S. Kaczmarz and H. Steinhaus Theorie der orthogonalreihen, Monatsh. Math. 4 (1935).

5. D. Menchoff Sur les séries de fonctions orthogonales, Fund. Math. IV (1923), 82-105

6. F. Moricz On the Cesàro means of orthogonal sequences of random variables, Ann. Probab. 11 (1983), 827-832.

7. _ Strong laws of large numbers for orthogonal sequences of random variables, Limit Theorems in Probability and Statistics (Veszprem, Hungary), Colloq. Math. Soc. János Bolyai, vol. 36, 1982, pp. 807-821.

8. H. Rademacher Einige Sätze über Reihen von Allgemeinen Orthogonalfunktionen, Math. Ann. 87 (1922), 112-138.

Institute of Mathematics, University of Lódź, ul. Stefana Banacha 22, 90-238 Lódź, Poland 